\title{
Programmed Intermittent Epidural Bolus (PIEB) versus Patient Controlled Epidural Analgesia (PCEA) plus background infusion for labour analgesia. A randomised double blind clinical trial
}

Diez-Picazo L.D., Guasch E., Alvar E., Diez J*., Schiraldi R., Gilsanz F.

Dept. of Anaesthesiology \& Intensive Care and *Research and Development Department, University Hospital La Paz, Madrid, Spain

\section{BACKGROUND:}

$\checkmark$ Epidural analgesia (EA) and combined spinal-epidural (CSE) are commonly used for labour.

$\checkmark$ PCEA and PIEB are the favourite analgesic regimens during labour.

$\checkmark$ Breakthrough pain (BP) is even today a frequent complain in labour and the ideal neuraxial block (NB) and analgesic regimen are currently unclear.

\section{GOALS:}

* To compare PIEB versus PCEA measuring the incidence of breakthrough pain (BP)

* To evaluate the role of the neuraxial block (EA versus $\mathrm{CSE}$ ) in $\mathrm{BP}$ incidence.

\section{RESULTS:}

INCIDENCE OF BREAKTHROUGH PAIN (Figure 2): $>65 \%$ patients in PIEB groups had no BP episodes. $>13 \%$ patients in PIEB groups had no pain $(p<0.01)$. $>$ No difference of BP incidence between EA and CSE.

\section{METHODS:}

D/nclusion Criteria: Nulliparous (18-40yr), singleton with cervical dilation $\leqq 4 \mathrm{~cm}$.

Excluded: Contraindicated NB, systemic disease or lack of consent. $\square$ Assignment by a computer-generated random-number sequence DPatients and caregivers were blind to group assignment and analgesic regimen (figure 1 ).

All patients received a $5 \mathrm{ml} / \mathrm{h}$ background epidural infusion $(0.125 \%$ L-bupivacaine $+1.45 \mathrm{mcg} / \mathrm{ml}$ fentanyl).

$\square$ PIEB groups received $10 \mathrm{ml}$ bolus per hour (20 min lock time with epidural top-ups).

Epidural top-ups as PCEA were set up to dispense $10 \mathrm{ml}$ boluses (20 min lockout interval).

$\square$ BP was defined as a visual analogue scale (VAS) $\geqq 4$.

Failed block: VAS $\geqq 4$ sixty minutes after technique or lateralization ( 3 dermatomes).

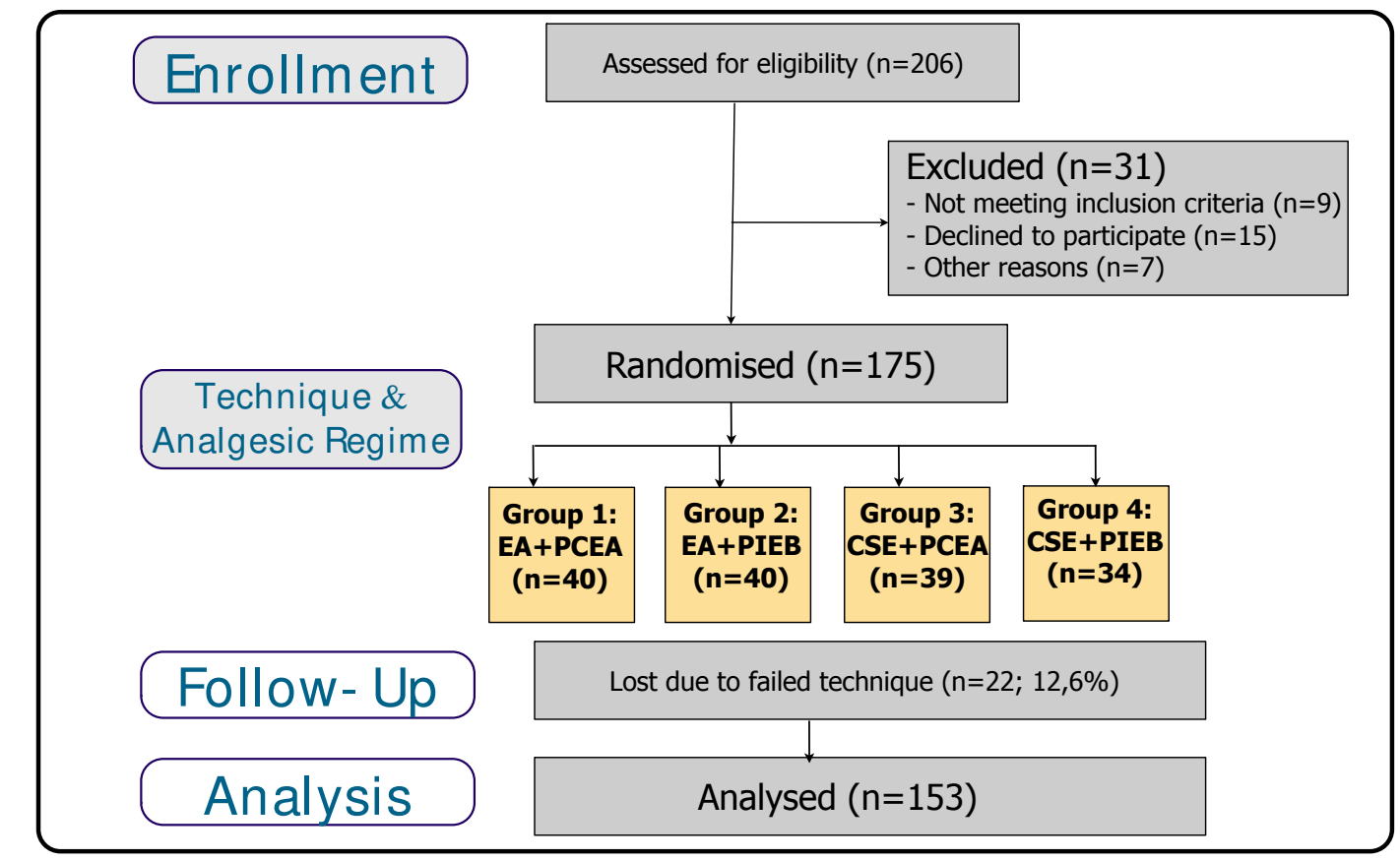

Figure 1: CONSORT flow chart

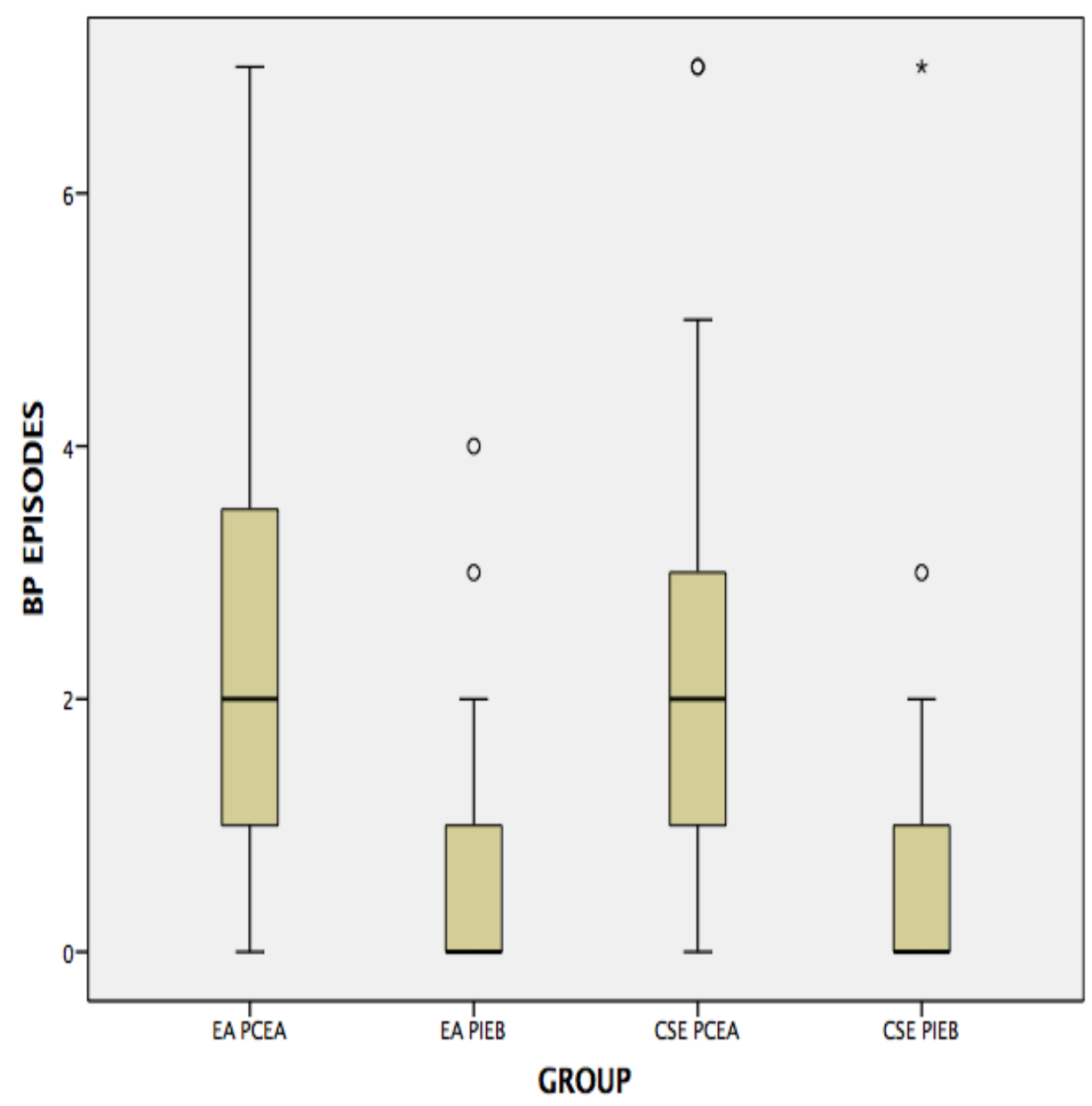

Figure 2: Incidence of breakthrough pain

EPIDURAL TOP-UPS (Figure 3):

$>$ Top-up volume was markedly lower in PIEB groups

$>$ Time to the first top-up was higher in PIEB:

- 214 vs 145 min after EA

- 317 vs 158 min after CSE $(p<0.01)$

$>$ No difference in top-up volume regarding NB.

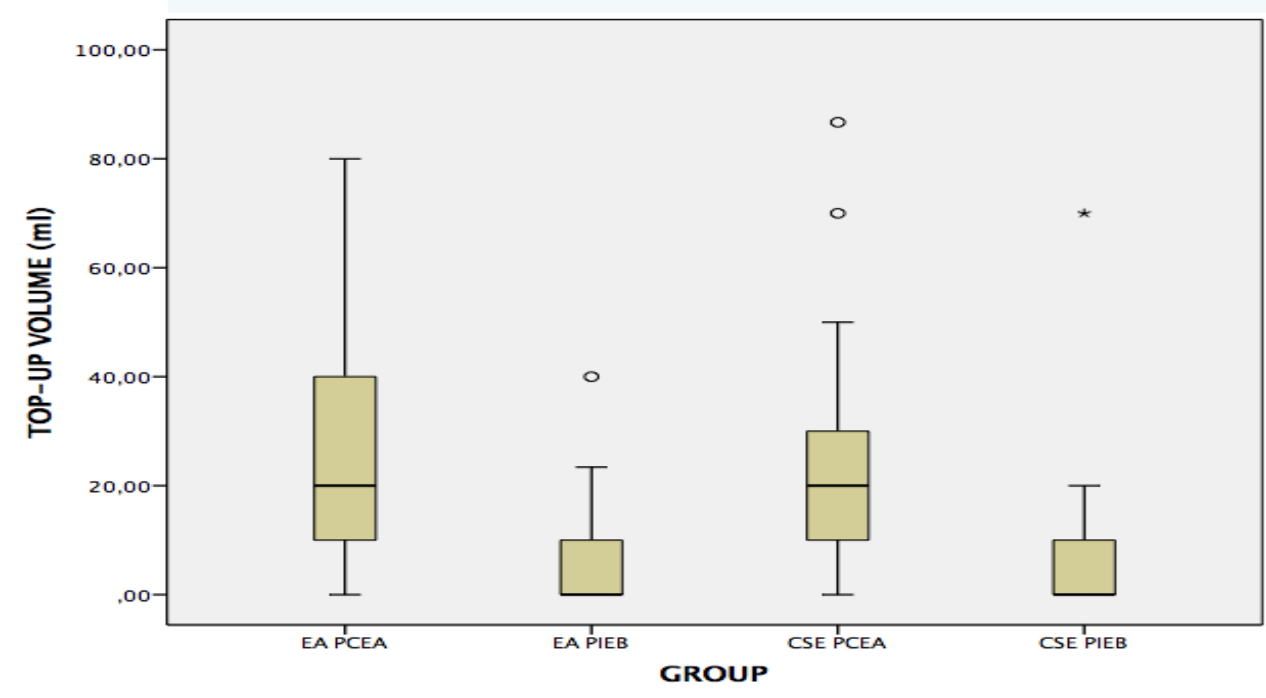

Figure 3: Epidural top-ups

\section{SAFETY RESULTS:}

$>$ No differences in mode of delivery among groups.

$>$ Similar delivery phases and adverse effects.

$>$ Analgesia lateralization was reduced by $\mathrm{PIEB}$ in EA groups ( $28 \%$ PCEA vs $12 \%$ PIEB; $p<0.01)$.

\section{CONCLUSIONS:}

1.- PIEB reduced the incidence of breakthrough pain in labour compared to PCEA.

2.- Neuraxial block (EA vs CSE) did not affect the incidence of BP during labour. 